\title{
Emergent Ferromagnetism with Fermi-Liquid Behavior in Proton Intercalated $\mathrm{CaRuO}_{3}$
}

\author{
Shengchun Shen, ${ }^{1}$ Zhuolu Li, ${ }^{1}$ Zijun Tian, ${ }^{2}$ Weidong Luo®, ${ }^{2,3, *}$ Satoshi Okamoto $\odot^{4, \dagger}$ and Pu Yu $\odot^{1,5,6, \$}$ \\ ${ }^{1}$ State Key Laboratory of Low Dimensional Quantum Physics and Department of Physics, \\ Tsinghua University, Beijing 100084, China \\ ${ }^{2}$ Key Laboratory of Artificial Structures and Quantum Control, School of Physics and Astronomy, \\ Shanghai Jiao Tong University, Shanghai 200240, China \\ ${ }^{3}$ Institute of Natural Sciences, Shanghai Jiao Tong University, Shanghai 200240, China \\ ${ }^{4}$ Materials Science and Technology Division, Oak Ridge National Laboratory, \\ Oak Ridge, Tennessee 37831, USA \\ ${ }^{5}$ Frontier Science Center for Quantum Information, Beijing 100084, China \\ ${ }^{6}$ RIKEN Center for Emergent Matter Science (CEMS), Wako 351-198, Japan
}

(Received 9 September 2020; revised 9 March 2021; accepted 22 March 2021; published 21 April 2021)

\begin{abstract}
The evolution between Fermi-liquid and non-Fermi-liquid states in correlated electron systems has been a central subject in condensed matter physics because of the coupled intriguing magnetic and electronic states. An effective pathway to explore the nature of non-Fermi-liquid behavior is to approach its phase boundary. Here we report a crossover from non-Fermi-liquid to Fermi-liquid state in metallic $\mathrm{CaRuO}_{3}$ through ionic liquid gating induced protonation with electric field. This electronic transition subsequently triggers a reversible magnetic transition with the emergence of an exotic ferromagnetic state from this paramagnetic compound. Our theoretical analysis reveals that hydrogen incorporation plays a critical role in both the electronic and magnetic phase transitions via structural distortion and electron doping. These observations not only help understand the correlated magnetic and electronic transitions in perovskite ruthenate systems, but also provide novel pathways to design electronic phases in correlated materials.
\end{abstract}

DOI: $10.1103 /$ PhysRevX.11.021018

\section{INTRODUCTION}

Landau Fermi-liquid (FL) theory has been a successful model for describing the low-temperature electronic properties of metals [1]. However, recently there have appeared an increasing number of systems that challenge the concept of FL by showing anomalous properties with non-Fermiliquid (NFL) behavior [2-8]. How to understand the NFL behavior has been an essential topic in the study of strongly correlated systems. Among these systems, transition metal oxides have attracted particular interest due to their vastly unusual properties with notable NFL behavior [9-14], and the corresponding investigation is an important factor toward understanding their fascinating properties. For instance, near a magnetic transition, the associated order parameter fluctuations may lead to the breakdown of FL

\footnotetext{
*wdluo@sjtu.edu.cn

†okapon@ornl.gov

†yupu@mail.tsinghua.edu.cn
}

Published by the American Physical Society under the terms of the Creative Commons Attribution 4.0 International license. Further distribution of this work must maintain attribution to the author(s) and the published article's title, journal citation, and DOI.
Subject Areas: Condensed Matter Physics,

Magnetism, Materials Science and the emergence of NFL behavior [4,5,15]. Although extensive investigations have been carried out in heavy fermion compounds and metallic alloys [4-6], the correlation of NFL and magnetic phase transition in transition metal oxides remains rare $[11,12,16]$, which is likely due to the lack of effective pathways to obtain delicate manipulation of their magnetic and electronic states across these transitions.

Ruthenates represent an important family of complex oxides with a rich spectrum of properties, ranging from unconventional superconductivity to ferromagnetism [12,17-19]. Among this family, $\mathrm{CaRuO}_{3}$ has been extensively studied due to its unique nonmagnetic metallic state, as well as notable NFL behavior [16,20-22]. Interestingly, its isoelectronic $\mathrm{SrRuO}_{3}$ shows a ferromagnetic ground state with FL behavior [19], and such distinct magnetic states were recognized due to the change of crystalline structure $[23,24]$. The contrasting magnetic and electronic properties between $\mathrm{SrRuO}_{3}$ and $\mathrm{CaRuO}_{3}$ suggest an intimate correlation between the Fermi-liquid behavior and ferromagnetic state in those systems. Hence, $\mathrm{CaRuO}_{3}$ provides a great playground to explore the correlation between NFL behavior and magnetic phase transition if one can introduce the ferromagnetic state into this system. Chemical substitution with B site isovalent 
chemical ions was widely employed to manipulate the magnetic state in $\mathrm{CaRuO}_{3}$ in the past several decades with the aim to probe the magnetic and electronic transition simultaneously [25-31], which, however, generally leads to electron localization rather than itinerant ferromagnetism, and the resulted magnetism was likely attributed to the suppressed long-range magnetic ordering with local cluster [32-34]. While the $\mathrm{Sr}_{1-x} \mathrm{Ca}_{x} \mathrm{RuO}_{3}$ system undergoes a transition from ferromagnetic metal with FL behavior (FM FL) to paramagnetic metal with NFL behavior (PM NFL) with the increase of Ca concentration, the details close to the phase boundary differ substantially among previous reports limited by sample-to-sample variation [35-38]. Besides, previous theoretical work suggested that an itinerant ferromagnetic state can be potentially obtained from $\mathrm{CaRuO}_{3}$ through the epitaxial tensile strain [39], while the experimental results are controversial [36,40]. Therefore, it remains elusive whether a ferromagnetic FL metallic state could be obtained from $\mathrm{CaRuO}_{3}$, and if achievable how its FL behavior is correlated with the ferromagnetic state near the transition.

It is interesting to note that a recent study revealed a convenient method to control the magnetic state of $\mathrm{SrRuO}_{3}$ through the ionic liquid gating (ILG) induced proton intercalation (protonation) with the associated electron doping and structural deformation, which leads to a ferromagnetic to paramagnetic transition, while maintaining its robust FL behavior at low temperatures [41]. This study evokes immediately a series of interesting questions: whether the protonation process can lead to a novel magnetic state in $\mathrm{CaRuO}_{3}$, and how it would be coupled with the NFL behavior. In this paper, we demonstrate a reversible protonation of pristine $\mathrm{CaRuO}_{3}$ thin films through ILG. As a consequence, we observe an emergent ferromagnetic state as evidenced by the anomalous Hall effect. Through careful analysis of the temperature-dependent transport data, we reveal an interesting NFL to FL crossover as the precursor of the magnetic transition. We attribute this distinct PM NFL to FM FL transition to the protonation induced band structural modulation as well as suppressed electronic correlation.

\section{RESULTS}

\section{A. Proton intercalation in $\mathrm{CaRuO}_{3}$ film}

High-quality thin films of $\mathrm{CaRuO}_{3}$ were grown on (001) $\left(\mathrm{LaAlO}_{3}\right)_{0.3}-\left(\mathrm{SrAl}_{0.5} \mathrm{Ta}_{0.5} \mathrm{O}_{3}\right)_{0.7}$ (LSAT) substrates by a pulsed laser deposition system. Our samples show fully coherent epitaxial nature due to the relatively small lattice mismatch between sample and substrate. Figure 1(a) shows a schematic drawing of the ILG induced proton intercalation into the sample, in which protons are produced through the electrolysis of residual water within the ionic liquid
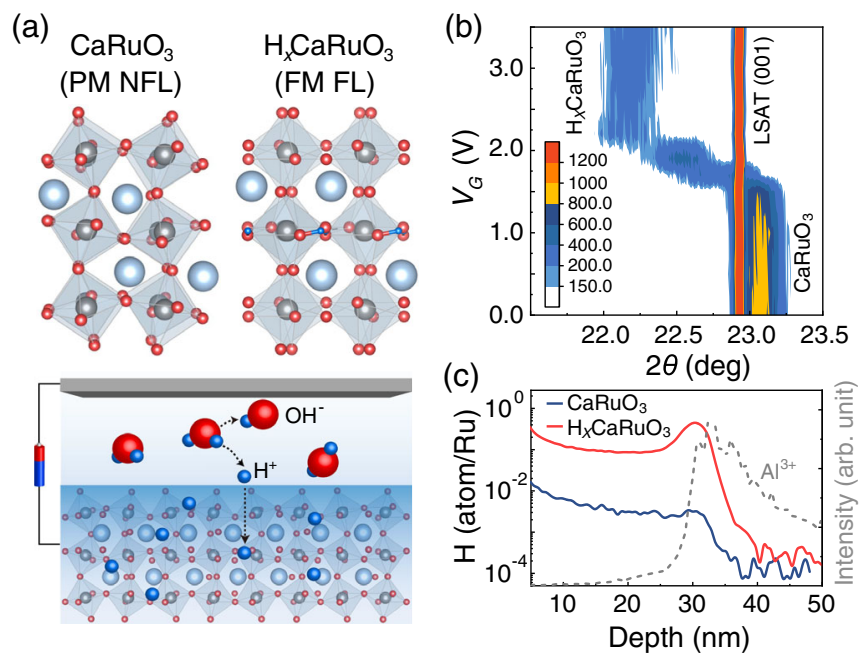

FIG. 1. Structural transformation of $\mathrm{CaRuO}_{3}$ through ionic liquid gating induced protonation. (a) Schematic illustration of proton intercalated induced phase transformation in $\mathrm{CaRuO}_{3}$ through ionic liquid gating (ILG). The upper panel shows a paramagnetic non-Fermi-liquid (PM NFL) metallic state in $\mathrm{CaRuO}_{3}$ to ferromagnetic Fermi-liquid behavior (FM FL) in $\mathrm{H}_{x} \mathrm{CaRuO}_{3}(x=0.5)$. The optimized crystalline structures were obtained from DFT calculations. The lower panel shows a schematic for the protonation process during the ILG. (b) In situ XRD $\theta-2 \theta$ scans around (001) peak of $\mathrm{CaRuO}_{3}$ as a function of gating voltage $\left(V_{G}\right)$. The peak position shifts gradually toward lower angle (from $23.07^{\circ}$ to $22.17^{\circ}$ ) as the $V_{G}$ increases, corresponding to a distinct lattice expansion along out of plane for up to $4.1 \%$. (c) Measured residual hydrogen concentration through ex situ SIMS on both pristine $\mathrm{CaRuO}_{3}$ and proton intercalated $\mathrm{H}_{x} \mathrm{CaRuO}_{3}$ (gated at $3.5 \mathrm{~V}$ ) samples. A referenced $\mathrm{Al}^{3+}$ signature was also shown to highlight the interface position between film and substrate.

and then inserted into the sample with the application of positive voltage across the gating electrode and sample [41-43]. To maintain charge neutrality, electrons are injected from the counter electrode into sample, completing the protonation process. With this setup, we first carried out in situ $\mathrm{x}$-ray diffraction (XRD) measurements during the ILG of $\mathrm{CaRuO}_{3}$ thin films. As shown in Fig. 1(b), with increasing gate voltage $\left(V_{G}\right)$, we observe a clear shift of the $\mathrm{CaRuO}_{3}$ (001) diffraction peak toward lower angles when $V_{G}$ is larger than $1.5 \mathrm{~V}$, and such a critical voltage is consistent with the previous study of $\mathrm{SrRuO}_{3}$, and is attributed to the water electrolysis process [41]. The $c$ lattice constant changes gradually from the pristine state and is eventually saturated with expansion of $\sim 4.1 \%$, providing the opportunity to manipulate continuously the electronic state of $\mathrm{CaRuO}_{3}$. It is important to note that the in-plane epitaxial strain remains unchanged throughout the phase transformation as evidenced by in situ reciprocal space mapping measurements (Fig. S1 [44]). Similar to the previous study of $\mathrm{SrRuO}_{3}$, the structural transformation is also volatile, i.e., the crystal structure returns back to its 
pristine state when the gate voltage is turned off. The slight offset of the XRD peak [as shown in Fig. S1(a) [44] ] is attributed to a small amount of residual hydrogen within the sample, as supported by our ex situ SIMS measurements on gated samples [Fig. 1(c)], in which about $10 \%$ hydrogen is observed in the postgated $\mathrm{H}_{x} \mathrm{CaRuO}_{3}$.

\section{B. Proton intercalation induced magnetic phase transition}

To trace the evolution of magnetic and electronic states in $\mathrm{CaRuO}_{3}$ thin films through protonation, we performed in situ transport measurements during ILG (see methods in Supplemental Material [44]). Figure 2(a) shows the temperature-dependent resistivity $\rho_{x x}(T)$ at different $V_{G}$. At lower $V_{G}$, the resistivity remains almost unchanged, while when $V_{G}$ is above $1.5 \mathrm{~V}$, the resistivity is slightly enhanced, which is attributed to the local lattice distortion induced by the protonation. With the observation of robust metallic state, we then study its magnetic state through the anomalous Hall effect (AHE) measurements [45]. For pristine $\mathrm{CaRuO}_{3}$ film, the Hall resistivity shows a linear dependence on the magnetic field [Fig. 2(b)], indicating a nonmagnetic state. Surprisingly, the gated samples (e.g., $V_{G}=2.5 \mathrm{~V}$ ) exhibit a distinct AHE hysteresis loop with nonzero remnant Hall resistivity at zero magnetic field, which signals an emergent ferromagnetic state through the ILG. Additionally, the magnetic field angle dependence of Hall resistance and longitudinal resistance suggests the magnetic easy axis in ferromagnetic $\mathrm{H}_{x} \mathrm{CaRuO}_{3}$ is along the out-of-plane direction (Fig. S3 [44]). It is important to note that the emergence of ferromagnetism by the protonation is exactly opposite to that of $\mathrm{SrRuO}_{3}$, in which the AHE, i.e., ferromagnetism, is totally suppressed through the protonation [41]. Furthermore, although the well-defined AHE hysteresis signals an emergent remnant ferromagnetization in proton intercalated $\mathrm{CaRuO}_{3}$, its actual spin configuration remains undetermined and is left for future investigations.

To trace the evolution of the ferromagnetic state in $\mathrm{H}_{x} \mathrm{CaRuO}_{3}$, we then systematically measured the temperature-dependent Hall resistivity as a function of $V_{G}$ (Fig. S2 [44]). Figure 2(c) summarizes the temperaturedependent AHE resistivity $\rho_{X Y}^{\mathrm{AHE}}$ at different $V_{G}$, in which $\rho_{X Y}^{\mathrm{AHE}}$ is defined as the saturated AHE component, as shown in the inset of Fig. 2(c). The systematic measurements reveal that the AHE (i.e., ferromagnetism) is observed with $V_{G}$ above $1.5 \mathrm{~V}$, with the maximum transition temperature around $20 \mathrm{~K}$. More importantly, the $V_{G}$ dependence of AHE is nonmonotonic, exhibiting a dome shape with increasing $V_{G}$ [Fig. 2(d)]. Furthermore, the extracted carrier concentration (hole type) increases by almost one order of magnitude at the initial stage of ILG and then decreases gradually for higher $V_{G}$ [Fig. 2(d)]. The notable similarity between the $V_{G}$-dependent carrier concentration and anomalous Hall resistivity suggests that the emergence
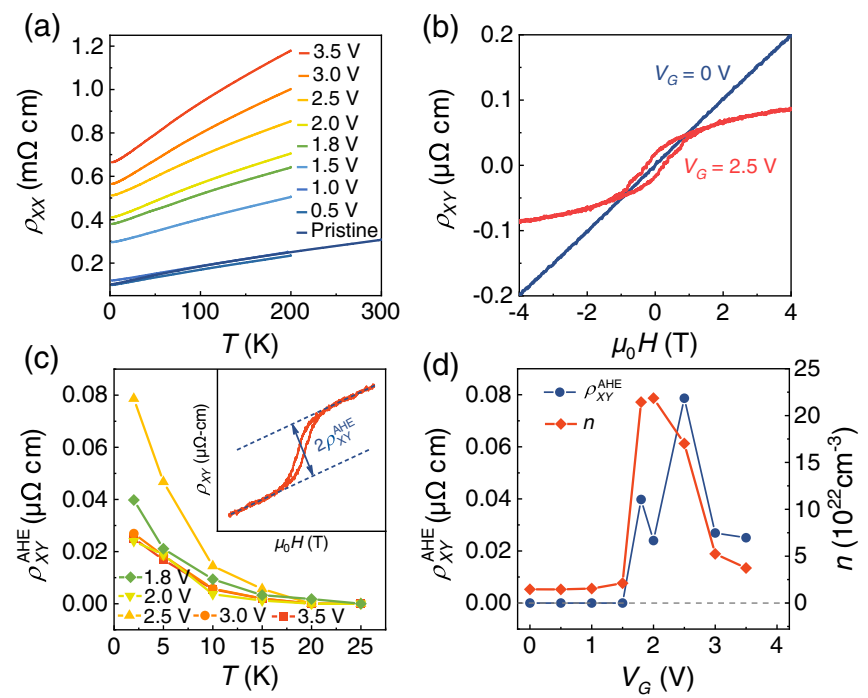

FIG. 2. Emergent ferromagnetism in proton intercalated $\mathrm{CaRuO}_{3}$ thin films. (a) In situ temperature dependence of resistivity at different $V_{G}$. (b) Magnetic field dependent Hall resistivity at $2 \mathrm{~K}$ for pristine and gated $\left(V_{G}=2.5 \mathrm{~V}\right)$ states, respectively. (c) Temperature-dependent anomalous Hall resistivity $\rho_{X Y}^{\mathrm{AHE}}$ at different $V_{G}$. The inset illustrates the method to obtain the $\rho_{X Y}^{\mathrm{AHE}}$ from a representative Hall loop $\left(V_{G}=2.0 \mathrm{~V}\right.$ and $T=2 \mathrm{~K}$ ). (d) Summary of $\rho_{X Y}^{\mathrm{AHE}}$ and carrier density ( $n$, extracted from linear part of Hall signal at high-magnetic-field region) as a function of $V_{G}$ at $2 \mathrm{~K}$.

of ferromagnetism in $\mathrm{H}_{x} \mathrm{CaRuO}_{3}$ is correlated with the electronic state. The discrepancy between the increase of hole concentration and hydrogen intercalation induced electron doping indicates that the Fermi surface of the sample is composed of both electronlike and holelike pockets.

\section{Proton intercalation induced NFL to FL transition}

With this established magnetic phase diagram, we then further investigate the evolution of NFL through the ILG. Generally, NFL manifests itself into different power-law behavior of physical quantities from those of a FL. For instance, the temperature-dependent resistivity can be fitted with $\rho \sim T^{\alpha}$, where $\alpha=2$ for FL and typically $\alpha<2$ for NFL $[2,3,5,16,20]$. To explore the potential variance in electronic behavior of the ILG sample, we quantitatively analyze the diagonal resistivity $\rho_{x x}(T)$ by fitting the curves with the empirical relation $\rho \sim T^{\alpha}$ at low temperatures (Fig. S4 [44]). Intriguingly, the exponent $\alpha$ clearly shows two regimes. As shown in Fig. 3(a), the pristine film and gated samples with $V_{G}$ of 0.5 and $1.0 \mathrm{~V}$ show an obvious power-law behavior with $\alpha \sim 3 / 2$ at low temperatures, and such a NFL behavior is typically attributed to the diffusive electron motion induced by strong interactions between itinerant electrons and the critically damped long-wavelength magnons in quantum critical point systems $[20,46]$. With increasing $V_{G}$ at $1.5 \mathrm{~V}$ and above, $\alpha$ changes from $\sim 3 / 2$ to $\sim 2$ [Fig. 3(b)], indicating an electronic transition 

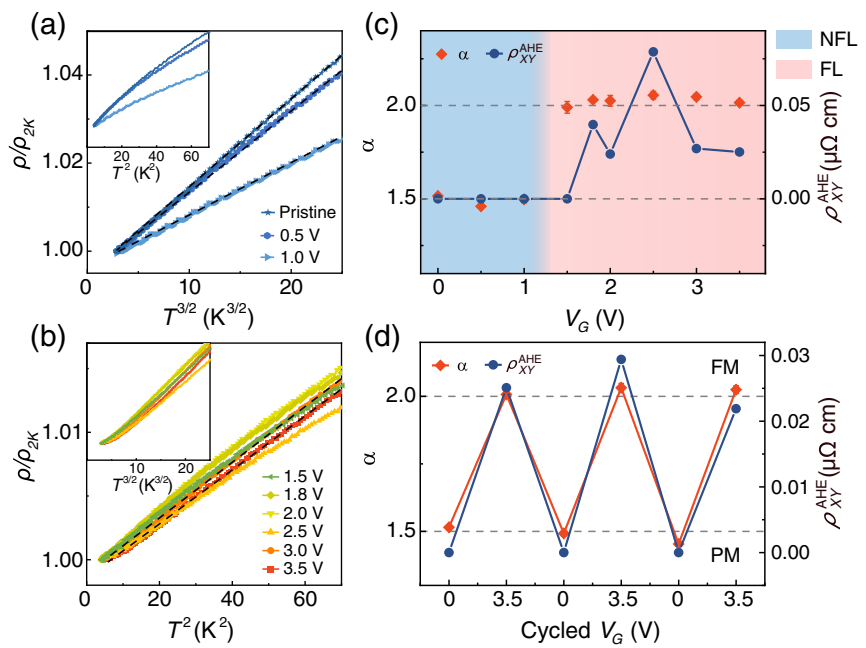

FIG. 3. Evolution from non-Fermi-liquid to Fermi-liquid behavior accompanied by magnetic transition. Normalized longitude resistivity $\rho_{x x /} \rho_{2 K}$ as a function of (a) $T^{3 / 2}$ and (b) $T^{2}$ at low-temperature region with different $V_{G}$. Insets show the deviation from $T^{2}\left(T^{3 / 2}\right)$ for pristine (gated at $V_{G}>1.0 \mathrm{~V}$ ) states for comparison. The dashed lines are guides to the eyes. (c) Obtained fitting exponent $\alpha$ as a function of $V_{G}$ for $\rho(T) \sim T^{\alpha}$, forming two regions labeled by NFL and FL. The $V_{G}$ dependence of $\rho_{X Y}^{\mathrm{AHE}}$ is also shown. (d) Reversible evolution of fitting exponent $\alpha$ and $\rho_{X Y}^{\mathrm{AHE}}$, as $V_{G}$ cycled between 0 and $3.5 \mathrm{~V}$.

from a NFL to a FL. It is noteworthy that the Hall measurements reveal that the ferromagnetic state (with $\rho_{X Y}^{\mathrm{AHE}} \neq 0$ ) emerges only at $V_{G}>1.5 \mathrm{~V}$ [Fig. 3(c) and Fig. S2 [44]), which clearly indicates that the ferromagnetism emerges subsequently after the FL, rather than emerging simultaneously. This further suggests that the FL appears as a precursor of the ferromagnetism in a $\mathrm{CaRuO}_{3}$ system, which is consistent with the fact that the $\mathrm{FL}$ is robust through the magnetic transition in $\mathrm{SrRuO}_{3}$ [39]. Furthermore, it is also found that this magnetic and electronic transition can be induced reversibly with the application of gating voltage, showing an exotic magnetoelectric coupling. More specifically, when $V_{G}$ is cycled between 0 and $3.5 \mathrm{~V}$, the on-off switching of ferromagnetic states can be realized, and the electronic behavior translates between NFL and FL as well, as shown in Fig. 3(d) and Fig. S5 [44].

\section{Mechanism of proton intercalation induced phase transitions}

To gain insight into the emergent ferromagnetism and $\mathrm{FL}$ behavior in $\mathrm{H}_{x} \mathrm{CaRuO}_{3}$, we carried out density functional theory (DFT) calculations, and then integrated them with dynamical mean-field theory (DMFT) calculations, i.e., DFT + DMFT. The details are presented in Supplemental Material [44]. The optimized crystalline structure of $\mathrm{H}_{x} \mathrm{CaRuO}_{3}(x=0.5)$ is shown in Fig. 1(a), in which the intercalated proton ions prefer to form bonding with equatorial oxygen ions in $\mathrm{RuO}_{6}$ octahedra. A systematic theoretical calculation reveals that along the protonation, the crystalline structure process an enlarged $\mathrm{Ru}-\mathrm{O}-\mathrm{Ru}$ bonding angle and expanded crystalline lattice with larger $c / a$ ratio (tetragonality) (Fig. S6 [44]). Consequently, such structure modification results in the splitting of degenerate $\mathrm{Ru} t_{2 g}$ bands as well as narrower bandwidth (Fig. S6 [44]). Furthermore, with increasing intercalated proton concentration, the electron band spectra weight shifts gradually toward lower energy, due to the electron band filling, in which the $\mathrm{Ru}^{4+}$ turns into $\mathrm{Ru}^{3+}$ for the fully protonated case. More importantly, the proton intercalation leads to the characteristic modulation of density of states (DOS) at the Fermi level, as revealed in Figs. S7 and S8 with DFT and DFT + DMFT methods, respectively [44]. The pristine $\mathrm{CaRuO}_{3}$ shows suppressed spectral weights at Fermi level $(E=0)$ [Figs. S7(a) and S8(a) [44]], which is consistent with earlier theoretical study [23] and accounts for the paramagnetic ground state following the Stoner criteria. By contrast, the proton intercalation process leads to a pronounced enhancement of DOS in the van Hove singularity around the Fermi level [Figs. S7(b)-S7(d),S8(b)-S8(d) [44]]. Such peaked DOS could potentially have the Stoner instability, leading to ferromagnetic ordering in itinerant electron system $[47,48]$. As expected, ferromagnetism is found to be stabilized in DFT + DMFT for some $\mathrm{H}_{x} \mathrm{CaRuO}_{3}$ cases, e.g., $\mathrm{H}_{0.5} \mathrm{CaRuO}_{3}$ in Fig. 4(a) (Fig. S9 [44]). The relatively small difference between the minority spin and majority spin DOS suggests that $\mathrm{H}_{0.5} \mathrm{CaRuO}_{3}$ is a weak ferromagnet, which is consistent with our experimental observations of low Curie temperature and small AHE. In addition, the electronic behavior also changes with proton intercalation, as indicated by the imaginary part of the self-energy [Fig. 4(b) and Fig. S8 [44] ]. For pristine $\mathrm{CaRuO}_{3}$, it behaves as $\left(\omega_{n}\right)^{1 / 2}$, which indicates a nonFermi-liquid behavior due to the spin freezing [Fig. 4(b)] [22]. For $\mathrm{H}_{0.5} \mathrm{CaRuO}_{3}$, the frequency range of this $\left(\omega_{n}\right)^{1 / 2}$ behavior becomes narrower and the absolute value of the self-energy becomes much smaller than that of $\mathrm{CaRuO}_{3}$, suggesting it is more Fermi-liquid-like [Fig. 4(b)]. In accordance with our experimental observations, DFT + DMFT calculations also manifest that proton intercalation induces a magnetic phase transition following the evolution from NFL to FL behavior.

As mentioned above, the proton intercalation process could have two contributions to influence the magnetic and electronic states: (i) modifying band structure through lattice distortion and (ii) doping electrons. Therefore, to quantitatively trace the ferromagnetism caused by proton intercalation, we carried out DFT + DMFT calculations by changing the band structure for $\mathrm{H}_{x} \mathrm{CaRuO}_{3}$ and the $\mathrm{Ru} d$ electron number $N_{e}$ independently. One can see that magnetization $M$, which is defined by the difference between the up electron density and the down electron density, is nonzero and changes nonmonotonically with varying $N_{e}$ at fixed $x$ [Fig. 4(c)], suggesting that the ferromagnetism is correlated with the electron number $N_{e}$. M also changes dramatically by simply considering modified band structure induced by 

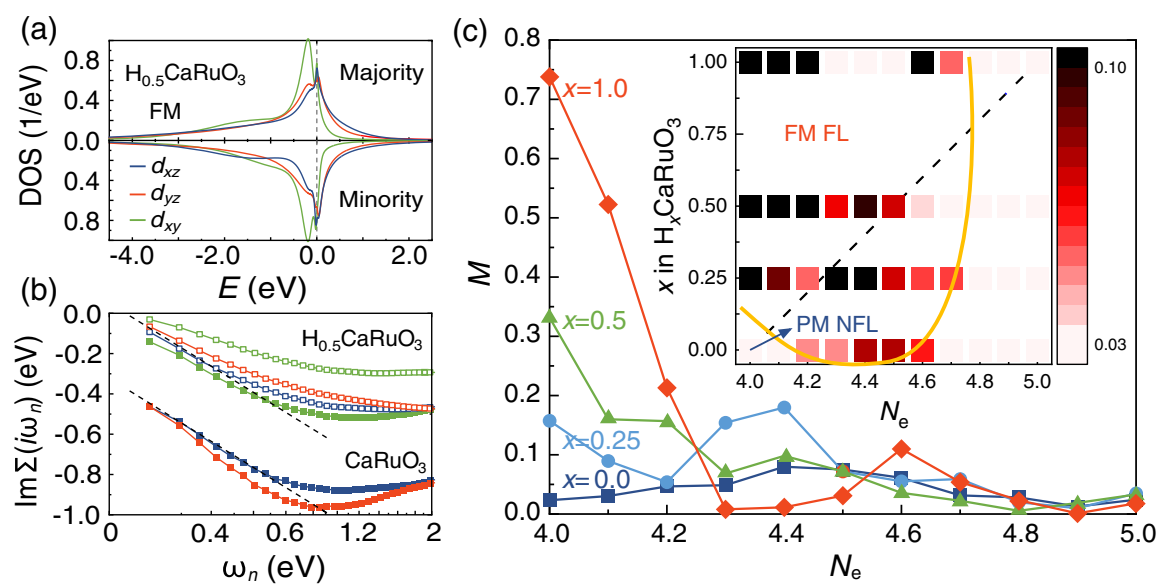

FIG. 4. Theoretically calculated phase diagram of proton intercalated $\mathrm{H}_{x} \mathrm{CaRuO}_{3}$. (a) Spin polarized density of states (DOS) of $\mathrm{H}_{0.5} \mathrm{CaRuO}_{3}$ obtained through DFT + DMFT calculations. The vertical dashed line marks the Fermi level which is taken to be the origin of the horizontal axis. (b) Imaginary part of the self-energy as a function of Matsubara frequency $\omega_{n}$ for $\mathrm{CaRuO}_{3}$ (filled symbols) and $\mathrm{H}_{0.5} \mathrm{CaRuO}_{3}$ (open symbols). A paramagnetic state is considered for both cases. The color codes used denote different orbitals in the same way as (a), and the dashed lines indicate $\left(\omega_{n}\right)^{1 / 2}$ behavior. (c) Calculated Ru magnetic moment $M$ as a function of Ru $d$ electron number $\left(N_{e}\right)$ for pristine $\mathrm{CaRuO}_{3}$ (blue square), $\mathrm{H}_{0.25} \mathrm{CaRuO}_{3}$ (light blue circle), $\mathrm{H}_{0.5} \mathrm{CaRuO}_{3}$ (green triangle), and $\mathrm{HCaRuO}$ (red diamond), respectively. The inset shows a summarized intensity map of magnetization $(M)$ as a function of both $N_{e}$ and $x$, in which the color bar denotes $M$. The yellow line shows guideline for the boundary between ferromagnetism (FM) and paramagnetism (PM), while the dashed black line represents the actual protonation effect with simultaneous structural expansion and electron doping (as $\left.N_{e}=4+x\right)$.

different hydrogen concentrations with fixed $N_{e}=4$, indicating ferromagnetism is also correlated with the structural distortion. The inset of Fig. 4(c) shows a resulting theoretical phase diagram of the $\mathrm{H}_{x} \mathrm{CaRuO}_{3}$ system as a function of hydrogen concentration $x$ and electron number $N_{e}$. Ferromagnetic ordering is pronounced at the intermediate $x$ and $N_{e}$ as indicated by a yellow line, accompanied with Fermi-liquid behavior emerging from a paramagnetic non-Fermi-liquid state. In the actual experimental situation, the electron number depends on the hydrogen concentration as $N_{e}=4+x$. As shown as a dashed line in the inset of Fig. 4(c), this $N_{e}$ goes across the ferromagnetic regime extending from the upper left-hand corner to the lower right-hand corner, resulting from the combination of structural distortion, which pushes down the position of the van Hove singularity, and electron doping, which pushes up the Fermi level. Thus, the theoretical phase diagram is qualitatively consistent with the dome-shaped AHE signal we observed. It is interesting to note that the largest $M$ is found at $N_{e}=4$ with the fully proton intercalated $\mathrm{HCaRuO}_{3}$ structure (Fig. S10 [44]). This suggests that stronger ferromagnetism could be achieved by doping holes into the fully proton intercalated $\mathrm{HCaRuO}_{3}$ or by inducing the similar structural distortion as $\mathrm{HCaRuO}_{3}$ without changing the nominal $\mathrm{Ru}$ valence by, for example, He implantation [49].

\section{DISCUSSION}

Because $d$ orbitals of $4 d$ transition metal ions are typically more extended than those of $3 d$ transition metal ions, the $p$ - $d$ hybridization of $\mathrm{O}-\mathrm{Ru}$ bond is sensitive to the lattice distortion. Therefore, the subtle balance and competition between strong $p$ - $d$ hybridization, electronlattice coupling, as well as the Coulomb interaction in $4 d$ transition metal oxides can lead to dramatic evolution of the corresponding electronic and magnetic states. Our current results strongly suggest that the intercalated hydrogen atoms can readily tip the balance between different phases, leading to drastic changes in magnetic and electronic properties. As shown in the above observations, the protonation in $\mathrm{CaRuO}_{3}$ leads to a magnetic phase transition, which is correlated with both lattice distortion and electron doping induced by intercalated protons. Additionally, both experimental and theoretical results suggest that the Fermi-liquid transition forms a precursor of the induced ferromagnetism in a proton intercalated $\mathrm{CaRuO}_{3}$ system. It is interesting to note that our previous work on proton intercalated $\mathrm{SrRuO}_{3}$ suggests the FL behavior is maintained throughout the FM to PM magnetic transition [41]. These results are clearly contrary to a general consensus of FL instability at magnetic phase transition [5], in which the ferromagnetism and FL behavior is decoupled in $\mathrm{Ca}(\mathrm{Sr}) \mathrm{RuO}_{3}$ systems. The NFL behavior is driven by strong electronic correlations that freeze local spin moments [22], the protonation could weaken the electronic correlations by changing $d$ electron density away from an integer value 4 . It is clear that the FL behavior represents the dominant factor for the emergent ferromagnetism in proton intercalated $\mathrm{CaRuO}_{3}$, due to the fact that the electron correlation modified by protonation plays a 
critical role for the magnetic transition. The whole process of proton intercalation $\mathrm{CaRuO}_{3}$ could be understood as follows: The protonation process first leads to the breakdown of NFL in $\mathrm{H}_{x} \mathrm{CaRuO}_{3}$ with relatively small amount of $\mathrm{H}$ concentration due to the suppressed electron correlation. Then with further protonation, a ferromagnetic ground state emerges due to Stoner instability caused by modified band structure and suppressed electron correlation. While for $\mathrm{SrRuO}_{3}$, the FL behavior is preserved across the FM to PM transition through protonation because it is a weakly correlated system.

In summary, we demonstrated that an itinerant ferromagnetic state can be introduced in paramagnetic $\mathrm{CaRuO}_{3}$ via ILG induced protonation. As a precursor of this magnetic phase transition, the NFL to FL transition in electronic behavior appears due to the suppressed electronic correlation. More importantly, these transitions can be manipulated reversibly in an electric manner, suggesting an exotic magnetoelectric coupling effect. Our work not only leads to a further understanding of the itinerant ferromagnetism as well as NFL and FL behaviors in $(\mathrm{Ca}, \mathrm{Sr}) \mathrm{RuO}_{3}$ systems, but also highlights that the electrically controllable protonation could serve as an effective pathway to manipulate the electronic and magnetic phase transitions in strongly correlated electron systems. It could trigger general research interest to design the phase diagram of ruthenate systems, and a rich spectrum of novel properties could emerge.

\section{ACKNOWLEDGMENTS}

This work was financially supported by the Basic Science Center Program of NSFC (Grant No. 51788104); NSFC (Grants No. 51872155, No. U1632272, 11521404 , and No. 11904196); the National Basic Research Program of China (Grant No. 2016YFA0301004); the Tsinghua University Initial Science Research Program (20203080003) and the Beijing Advanced Innovation Center for Future Chip (ICFC); and the Engineering and Physical Sciences Research Council (Grant No. EP/ N016718/1). The research by S. O. was supported by the U.S. Department of Energy, Office of Science, Basic Energy Sciences, Materials Sciences and Engineering Division. This research used resources of the Compute and Data Environment for Science (CADES) at the Oak Ridge National Laboratory, which is supported by the Office of Science of the U.S. Department of Energy. Firstprinciples DFT calculations were performed at the HPC of Shanghai Jiao Tong University. P. Y. and S. S. conceived the project and designed the experiments. S. S. conducted the transport measurements and analyzed all data. Z. L. and S. S. grew the samples and performed XRD measurements. Z. T., W. L., and S.O. carried out DFT + DMFT calculations. S. S., S. O., and P. Y. wrote the manuscript, and all authors commented on the paper.
[1] L. D. Landau, The Theory of a Fermi Liquid, Sov. Phys. JETP 3, 920 (1957), http://www.jetp.ac.ru/files/Landau1en .pdf.

[2] C. Pfleiderer, S. R. Julian, and G. G. Lonzarich, Non-FermiLiquid Nature of the Normal State of Itinerant-Electron Ferromagnets, Nature (London) 414, 427 (2001).

[3] N. Doiron-Leyraud, I. R. Walker, L. Taillefer, M. J. Steiner, S. R. Julian, and G. G. Lonzarich, Fermi-Liquid Breakdown in the Paramagnetic Phase of a Pure Metal, Nature (London) 425, 595 (2003).

[4] G. R. Stewart, Non-Fermi-Liquid Behavior in $d$ - and f-Electron Metals, Rev. Mod. Phys. 73, 797 (2001).

[5] H. V. Löhneysen, A. Rosch, M. Vojta, and P. Wölfle, FermiLiquid Instabilities at Magnetic Quantum Phase Transitions, Rev. Mod. Phys. 79, 1015 (2007).

[6] S.-S. Lee, Recent Developments in Non-Fermi Liquid Theory, Annu. Rev. Condens. Matter Phys. 9, 227 (2018).

[7] R. Ritz, M. Halder, M. Wagner, C. Franz, A. Bauer, and C. Pfleiderer, Formation of a Topological Non-Fermi Liquid in MnSi, Nature (London) 497, 231 (2013).

[8] B. Shen et al., Strange-Metal Behaviour in a Pure Ferromagnetic Kondo Lattice, Nature (London) 579, 51 (2020).

[9] J. Orenstein and A. J. Millis, Advances in the Physics of High-Temperature Superconductivity, Science 288, 468 (2000).

[10] R. W. Hill, C. Proust, L. Taillefer, P. Fournier, and R. L. Greene, Breakdown of Fermi-Liquid Theory in a CopperOxide Superconductor, Nature (London) 414, 711 (2001).

[11] P. Khalifah, K. D. Nelson, R. Jin, Z. Q. Mao, Y. Liu, Q. Huang, X. P. A. Gao, A. P. Ramirez, and R. J. Cava, NonFermi-Liquid Behaviour in $\mathrm{La}_{4} \mathrm{Ru}_{6} \mathrm{O}_{19}$, Nature (London) 411, 669 (2001).

[12] S. A. Grigera et al., Magnetic Field-Tuned Quantum Criticality in the Metallic Ruthenate $\mathrm{Sr}_{3} \mathrm{Ru}_{2} \mathrm{O}_{7}$, Science 294, 329 (2001).

[13] E. Mikheev, A. J. Hauser, B. Himmetoglu, N. E. Moreno, A. Janotti, C. G. Van de Walle, and S. Stemmer, Tuning Bad Metal and Non-Fermi Liquid Behavior in a Mott Material: Rare-Earth Nickelate Thin Films, Sci. Adv. 1, e1500797 (2015).

[14] S. Stemmer and S. J. Allen, Non-Fermi Liquids in Oxide Heterostructures, Rep. Prog. Phys. 81, 062502 (2018).

[15] A. J. Schofield, Non-Fermi Liquids, Contemp. Phys. 40, 95 (1999).

[16] L. Klein, L. Antognazza, T. H. Geballe, M. R. Beasley, and A. Kapitulnik, Possible Non-Fermi-Liquid Behavior of $\mathrm{CaRuO}_{3}$, Phys. Rev. B 60, 1448 (1999).

[17] J. M. Longo, P. M. Raccah, and J. B. Goodenough, Magnetic Properties of $\mathrm{SrRuO}_{3}$ and $\mathrm{CaRuO}_{3}$, J. Appl. Phys. 39, 1327 (1968).

[18] Y. Maeno, H. Hashimoto, K. Yoshida, S. Nishizaki, T. Fujita, J. G. Bednorz, and F. Lichtenberg, Superconductivity in a Layered Perovskite without Copper, Nature (London) 372, 532 (1994).

[19] G. Koster, L. Klein, W. Siemons, G. Rijnders, J. S. Dodge, C.-B. Eom, D. H. A. Blank, and M. R. Beasley, Structure, Physical Properties, and Applications of $\mathrm{SrRuO}_{3}$ Thin Films, Rev. Mod. Phys. 84, 253 (2012).

[20] G. Cao, O. Korneta, S. Chikara, L. E. DeLong, and P. Schlottmann, Non-Fermi-Liquid Behavior in Single-Crystal 
$\mathrm{CaRuO}_{3}$ : Comparison to Ferromagnetic $\mathrm{SrRuO}_{3}$, Solid State Commun. 148, 305 (2008).

[21] M. S. Laad, I. Bradaric, and F. V. Kusmartsev, Orbital NonFermi-Liquid Behavior in Cubic Ruthenates, Phys. Rev. Lett. 100, 096402 (2008).

[22] P. Werner, E. Gull, M. Troyer, and A. J. Millis, Spin Freezing Transition and Non-Fermi-Liquid Self-Energy in a ThreeOrbital Model, Phys. Rev. Lett. 101, 166405 (2008).

[23] H. T. Dang, J. Mravlje, A. Georges, and A. J. Millis, Electronic Correlations, Magnetism, and Hund's Rule Coupling in the Ruthenium Perovskites $\mathrm{SrRuO}_{3}$ and $\mathrm{CaRuO}_{3}$, Phys. Rev. B 91, 195149 (2015).

[24] S. Middey, P. Mahadevan, and D. D. Sarma, Dependence of Magnetism on $\mathrm{GdFeO}_{3}$ Distortion in the $t_{2 g}$ System $\mathrm{ARuO}_{3}$ ( $A=\mathrm{Sr}, \mathrm{Ca})$, Phys. Rev. B 83, 014416 (2011).

[25] G. Cao, S. McCall, J. Bolivar, M. Shepard, F. Freibert, P. Henning, and J. E. Crow, Itinerant-to-Localized Electron Transition in $\mathrm{CaRu}_{1-x} \mathrm{Sn}_{x} \mathrm{O}_{3}$ and $\mathrm{SrRu}_{1-x} \mathrm{~Pb}_{x} \mathrm{O}_{3}$, Phys. Rev. B 54, 15144 (1996).

[26] G. Cao, F. Freibert, and J. E. Crow, Itinerant-to-Localized Electron Transition in Perovskite $\mathrm{CaRu}_{1-x} \mathrm{Rh}_{x} \mathrm{O}_{3}$, J. Appl. Phys. 81, 3884 (1997).

[27] T. He and R. J. Cava, Disorder-Induced Ferromagnetism in $\mathrm{CaRuO}_{3}$, Phys. Rev. B 63, 172403 (2001).

[28] T. He and R. J. Cava, The Effect of Ru-Site Dopants on the Magnetic Properties of $\mathrm{CaRuO}_{3}$, J. Phys. Condens. Matter 13, 8347 (2001).

[29] A. Maignan, C. Martin, M. Hervieu, and B. Raveau, Ferromagnetism and Metallicity in the $\mathrm{CaMn}_{1-x} \mathrm{Ru}_{x} \mathrm{O}_{3}$ Perovskites: A Highly Inhomogeneous System, Solid State Commun. 117, 377 (2001).

[30] A. Maignan, B. Raveau, V. Hardy, N. Barrier, and R. Retoux, Ferromagnetism Induced by Chromium Substitution in the $\mathrm{CaRuO}_{3}$ Perovskite, Phys. Rev. B 74, 024410 (2006).

[31] S. Mizusaki, T. Taniguchi, N. Okada, Y. Nagata, N. Hiraoka, T. Nagao, M. Itou, Y. Sakurai, T. C. Ozawa, and Y. Noro, Magnetic Properties of $\mathrm{CaRu}_{1-x} \mathrm{Fe}_{x} \mathrm{O}_{3}$, J. Appl. Phys. 99, 08 F703 (2006).

[32] V. Hardy, B. Raveau, R. Retoux, N. Barrier, and A. Maignan, Nature of the Ferromagnetism Induced by Nonmagnetic Substitutions on the Ru Site of $\mathrm{CaRuO}_{3}$, Phys. Rev. B 73, 094418 (2006).

[33] T. D. Yamamoto, R. Okazaki, H. Taniguchi, and I. Terasaki, Non-Uniform Magnetic System Driven by Non-Magnetic Ion Substitution in $\mathrm{CaRu}_{1-x} \mathrm{Sc}_{x} \mathrm{O}_{3}$ : Two-Component Analysis, J. Phys. Soc. Jpn. 84, 014708 (2015).

[34] I. Felner, U. Asaf, I. Nowik, and I. Bradaric, Magnetic and Mossbauer Studies of $\mathrm{CaRu}_{1-x} M_{x} \mathrm{O}_{3}(M=\mathrm{Ti}$ and $\mathrm{Fe})$, Phys. Rev. B 66, 054418 (2002).
[35] P. Khalifah, I. Ohkubo, H. M. Christen, and D. G. Mandrus, Evolution of Transport and Magnetic Properties Near the Ferromagnetic Quantum Critical Point in the Series $\mathrm{Ca}_{x} \mathrm{Sr}_{1-x} \mathrm{RuO}_{3}$, Phys. Rev. B 70, 134426 (2004).

[36] M. Schneider, V. Moshnyaga, and P. Gegenwart, Ferromagnetic Quantum Phase Transition in $\mathrm{Sr}_{1-x} \mathrm{Ca}_{x} \mathrm{RuO}_{3}$ Thin Films, Phys. Status Solidi B 247, 577 (2010).

[37] G. Cao, S. McCall, M. Shepard, J. E. Crow, and R. P. Guertin, Thermal, Magnetic, and Transport Properties of Single-Crystal $\mathrm{Sr}_{1-x} \mathrm{Ca}_{x} \mathrm{RuO}_{3}$, Phys. Rev. B 56, 321 (1997).

[38] K. Yoshimura, ${ }^{17} \mathrm{O}$ NMR Observation of Universal Behavior of Ferromagnetic Spin Fluctuations in the Itinerant Magnetic System $\mathrm{SrCaRuO}_{3}$, Phys. Rev. Lett. 83, 4397 (1999).

[39] A. T. Zayak, X. Huang, J. B. Neaton, and K. M. Rabe, Manipulating Magnetic Properties of $\mathrm{SrRuO}_{3}$ and $\mathrm{CaRuO}_{3}$ with Epitaxial and Uniaxial Strains, Phys. Rev. B 77, 214410 (2008).

[40] S. Tripathi, R. Rana, S. Kumar, P. Pandey, R. S. Singh, and D. S. Rana, Ferromagnetic $\mathrm{CaRuO}_{3}$, Sci. Rep. 4, 3877 (2014).

[41] Z. Li et al., Reversible Manipulation of the Magnetic State in $\mathrm{SrRuO}_{3}$ through Electric-Field Controlled Proton Evolution, Nat. Commun. 11, 184 (2020).

[42] N. Lu et al., Electric-Field Control of Tri-State Phase Transformation with a Selective Dual-Ion Switch, Nature (London) 546, 124 (2017).

[43] M. Wang et al., Electric-Field-Controlled Phase Transformation in $\mathrm{WO}_{3}$ Thin Films through Hydrogen Evolution, Adv. Mater. 29, 1703628 (2017).

[44] See Supplemental Material at http://link.aps.org/ supplemental/10.1103/PhysRevX.11.021018 for methods and supplemental figures.

[45] N. Nagaosa, J. Sinova, S. Onoda, A. H. MacDonald, and N. P. Ong, Anomalous Hall Effect, Rev. Mod. Phys. 82, 1539 (2010).

[46] C. Pfleiderer, M. Uhlarz, S. M. Hayden, R. Vollmer, H. v. Löhneysen, N. R. Bernhoeft, and G. G. Lonzarich, Coexistence of Superconductivity and Ferromagnetism in the d-Band Metal $\mathrm{ZrZn}_{2}$, Nature (London) 412, 58 (2001).

[47] I. I. Mazin and D. J. Singh, Electronic Structure and Magnetism in Ru-Based Perovskites, Phys. Rev. B 56, 2556 (1997).

[48] E. C. Stoner, XXXIII. Magnetism and Molecular Structure, London, Edinburgh, Dublin Philos. Mag. J. Sci. 3, 336 (1927).

[49] H. Guo et al., Strain Doping: Reversible Single-Axis Control of a Complex Oxide Lattice via Helium Implantation, Phys. Rev. Lett. 114, 256801 (2015). 